\title{
riccafd
}

Revista Iberoamericana de Ciencias de la Actividad Física y el Deporte

\section{RELACIÓN ENTRE CONDICIÓN FÍSICA Y SALUD MENTAL EN ESCOLARES DE PRIMARIA}

\section{RELATIONSHIP BETWEEN PHYSICAL FITNESS AND MENTAL HEATH IN PRIMARY SCHOOL CHILDREN}

\author{
Rosa-Guillamón, A y García-Cantó, $E^{2}$ \\ ${ }^{1}$ Rosa-Guillamón, Andrés. \\ Maestro de Educación Física en el C.B.M. Micaela Sanz Verde de Archena (Murcia, España). \\ andres.rosa@um.es \\ ${ }^{2}$ García-Cantó, Eliseo. \\ Profesor asociado de la Facultad de Educación de la Universidad de Murcia (Murcia, España). \\ Universidad de Murcia (Murcia, España). \\ eliseo.garcia@um.es \\ Agradecimientos \\ Los autores agradecen su colaboración a los colegios y escolares participantes en el estudio.
}

Código UNESCO: 2411.06 Fisiología del ejercicio

Clasificación Consejo de Europa: 6. Fisiología del ejercicio

Recibido el 10 de abril de 2016

Aceptado el 18 de junio de 2016

Correspondencia:

Andrés Rosa-Guillamón: andres.rosa@um.es

\section{RESUMEN}

Existen evidencias científicas de que la condición física es un marcador biológico de la salud. El objetivo fue analizar la relación de la condición física y el estado de peso con la salud mental en escolares de primaria del sureste español. Diseño descriptivo transversal donde se evaluó la condición física mediante la Batería ALPHA-fitness, a una muestra de 214 escolares entre 8 y 11 años. El índice de masa corporal fue categorizado siguiendo criterios estándar. La salud mental fue medida a través del General Health Questionnaire. El análisis de la varianza simple mostró que la condición física y el estado de peso no se asocian significativamente con la salud mental. Los resultados indican que la condición física y el estado de peso no constituyen elementos diferenciadores de la salud mental en escolares de 8 a 11 años del sureste español.

Palabras clave: salud, ejercicio físico, niños. 


\section{ABSTRACT}

There is scientific evidence that physical fitness is a biological marker of health. The aim of this study was to analyze the relationship between physical fitness and state weight with mental health in primary school children from the southeast of Span. An cross sectional design was implemented, evaluating handgrip strength, explosive lower body strength, aerobic capacity and speedagility through ALPHA-fitness test battery of a sample of 214 students between 8 and 11 years. The analysis of simple variance showed that physical fitness and state weight is not associated significantly with the mental health. The results indicate that physical fitness and state weight is not a differentiator of mental health in school children from the southeast of Spain.

Keywords: health, physical exercise, children.

\section{INTRODUCCIÓN}

La relación positiva entre actividad física y salud se encuentra bien establecida ${ }^{1}$. Se ha descrito que una actividad física habitual y con la intensidad adecuada contribuye a mejorar parámetros como el autoconcepto, estado de ánimo, felicidad o satisfacción vital ${ }^{2-5}$. Asimismo, se ha constatado que intervenciones basadas en la realización de ejercicio físico representan una de las estrategias más eficaces para prevenir las principales causas de morbimortalidad como la obesidad y el síndrome metabólico ${ }^{6}$.

Uno de los parámetros relacionados con el nivel de actividad física es la condición física (CF). La CF se define como la capacidad de una persona para la práctica física. El estado de CF representa una medida integrada de las funciones y estructuras que intervienen en la actividad física o ejercicio físicodeportivo. Estas funciones son: músculo-esquelética, cardio-respiratoria, endocrino-metabólica, hemato-circulatoria y psico-neurológica. La capacidad de realizar ejercicio físico depende de diversos componentes: aeróbico, músculo-esquelético, motor y morfológico ${ }^{7}$, e incluso coordinativo ${ }^{8}$.

Numerosos estudios muestran de manera inequívoca que el estado de CF constituye un excelente marcador biológico (y predictor) de la salud desde edades tempranas ${ }^{9-10 .}$

Recientes investigaciones han puesto de manifiesto el interés por analizar en escolares la relación de la CF y el estado de peso con indicadores positivos de la salud mental como calidad de vida, autoconcepto, felicidad o satisfacción vital $^{11-16}$.

Sin embargo, son escasos los trabajos realizados para analizar la relación de la CF y el estado de peso con parámetros negativos de la salud mental ${ }^{17}$. Algunos trabajos realizados con adultos y personas jóvenes han concluido que poseer un mejor nivel de CF se asocia a un estado positivo de salud mental ${ }^{18-19}$. 
En escolares, los estudios que han analizado la relación entre CF y salud mental se han centrado en la actividad física como variable mediadora en este proceso, siendo los resultados contradictorios ${ }^{20-24}$.

Algunos trabajos han encontrado efectos positivos en parámetros negativos de la salud mental tras la mejora en el nivel de CF mediante el seguimiento de un programa de ejercicio físico ${ }^{25-26}$. No obstante, en otros estudios se ha encontrado que las mejoras son leves o inexistentes ${ }^{27-28}$.

Teniendo en cuenta que hasta donde hemos constatado, no existen estudios que analicen esta temática en escolares de primaria, los objetivos del presente trabajo de investigación fueron: 1. abrir una nueva línea de investigación; y, 2. analizar la relación entre la condición física y el estado de peso con la salud mental en una muestra de escolares de 8 a 11 años del sureste español.

\section{MATERIAL Y MÉTODOS}

\section{Participantes}

Estudio descriptivo transversal con una muestra de 214 escolares del sureste español, en edades comprendidas entre 8-11 años (media \pm desviación estándar $=9,82 \pm 1,32$ años). Los escolares pertenecían a 5 centros públicos de Educación Primaria, y fueron seleccionados mediante muestreo no probabilístico intencional (Tabla 1). Se informó a los directores de la finalidad de la investigación, y se recibió por escrito consentimiento informado. Se planteó como criterio de exclusión la presencia de enfermedades mentales u óseas-articulares.

Todos los procedimientos siguieron las pautas establecidas en la Declaración de Helsinki (revisión de 2013), y la investigación respetó lo establecido por la Ley Orgánica de Protección de Datos (LOPD). Se recibió la aprobación del Comité de Bioética de la Universidad de Murcia (Murcia, España).

Tabla 1. Distribución de la muestra de estudio.

\begin{tabular}{|c|c|c|c|c|}
\hline \multirow[b]{2}{*}{ Edad (Años) } & \multicolumn{2}{|c|}{ Varones } & \multicolumn{2}{|c|}{ Mujeres } \\
\hline & $\mathrm{N}$ & $\%$ & $\mathrm{~N}$ & $\%$ \\
\hline 8 & 33 & 35,1 & 29 & 24,2 \\
\hline 9 & 4 & 4,3 & 15 & 12,5 \\
\hline 10 & 9 & 9,6 & 15 & 12,5 \\
\hline 11 & 48 & 51,1 & 61 & 50,8 \\
\hline Total & 94 & 100 & 120 & 100 \\
\hline
\end{tabular}

\section{Variables e instrumentos}

Condición física

Para medir la CF se seleccionaron diversas pruebas de la Batería ALPHA-Fitness basada en la evidencia, realizando modificaciones. Se omitieron los pliegues cutáneos por motivos de tiempo limitado, y se añadió el 
test de 4x10 metros que se plantea en la versión extendida, por la relación positiva entre velocidad-agilidad y salud ósea en escolares ${ }^{7}$. Durante todo el proceso se tuvo en cuenta el protocolo determinado en esta batería.

Se midió el peso corporal mediante una báscula electrónica (modelo 220, SECA, Hamburgo, Alemania). La talla fue medida con un estadiómetro (Holtain Ltd., Dyfed, UK). Se calculó el índice de masa corporal (IMC) definido como el peso en $\mathrm{kg}$ dividido por la talla en metros al cuadrado $\left(\mathrm{kg} / \mathrm{m}^{2}\right)$. Los escolares fueron categorizados siguiendo criterios estándar ${ }^{29}$.

La velocidad de desplazamiento-agilidad fue medida con el test de carrera $4 \times 10$ metros $^{30}$. Se utilizó como instrumento de medida un cronómetro profesional (HS-80TW-1EF, Casio, Tokio, Japan).

La fuerza de prensión manual fue medida a través de dinamometría manual mediante un dinamómetro digital con agarre ajustable (TKK 5041 Grip D, Takei, Tokio, Japan), y con una regla-tabla para adaptar la amplitud del agarre $^{31}$.

La potencia explosiva del tren inferior se valoró mediante salto longitudinal a pies juntos ${ }^{32}$. Se empleó para su medida una cinta métrica de PVC y fibra de vidrio (Modelo 74-Y100M, CST/Berger, Chicago, USA).

La capacidad aeróbica fue medida a través del test de Course-Navette ${ }^{33}$. Se anotó el último palier completado. Se empleó un equipo audio portátil (Behringer EPA40, Thomann, Burgebrach, Germany) y un dispositivo de memoria USB (Hayabusa, Toshiba, Tokio, Japan).

Las variables (velocidad-agilidad, fuerza de prensión manual, potencia explosiva del tren inferior y capacidad aeróbica) fueron transformadas dividiendo cada uno de los valores observados por el valor máximo de dicha variable. Posteriormente, se sumaron los valores de fuerza de prensión manual, potencia explosiva del tren inferior y capacidad aeróbica, y se restó el valor de velocidad-agilidad, dando como resultado una variable de CF global (zCF-ALPHA). Se categorizó a los escolares con menor CF global mCF-ALPHA $\left(X<P_{50}\right)$, y mayor CF global MCF-ALPHA $\left(X \geq P_{50}\right)$.

\section{Salud mental}

El cuestionario de salud mental (General Health Questionnaire; GHQ$12)^{34}$, fue elaborado para detectar trastornos psíquicos en un ámbito comunitario y en medios clínicos no psiquiátricos, y se centra en algunos componentes psicológicos que identifican una salud negativa: depresión, ansiedad, inadecuación social e hipocondría.

El GHQ-12 está formado por 12 ítems ( 6 sentencias positivas y 6 sentencias negativas). Los ítems son contestados a través de una escala tipo Likert con cuatro posibles respuestas, y con una puntuación dicotómica (0-0-1-1). La 
suma de estas puntuaciones da como resultado un valor global denominado GHQ-12, pudiendo clasificar a los sujetos en tres grupos:

- 0-4 puntos: ausencia de psicopatología.

- 5-6 puntos: sospecha de psicopatología subumbral.

- 7-12 puntos: indicativo de presencia de psicopatología.

El GHQ-12 presenta una buena fiabilidad en los diferentes estudios realizados con valores de alfa de Cronbach entre $0,76-0,86^{35-37}$. Los registros de validación del GHQ-12 realizado en 15 países del mundo son óptimos, con resultados de curva ROC entre 83 y $85^{35}$. En el presente estudio, el alfa de Cronbach fue de 0,718 .

\section{Procedimiento}

El trabajo fue realizado durante el curso académico (2014/15), en horario lectivo, y visitando los centros durante el mes de mayo de 2015.

El equipo de investigadores administró las pruebas a todos los participantes. Se recomendó a los padres que los escolares no realizasen actividades deportivas la tarde anterior, no variasen la alimentación habitual, y vistiesen ropa deportiva ligera. Primero, se realizaron las medidas de peso y talla y, posteriormente, se realizaron las medidas de CF. Se dejaron 5 minutos de descanso entre pruebas. Se realizó un calentamiento estándar de 8 minutos.

El cuestionario de salud mental (GHQ-12) se administró en grupos de 25 escolares, en un aula que permitía una separación física suficiente para mantener la privacidad, y con la presencia de los investigadores. Se rellenaron a primera hora de la mañana, siendo la duración media de 10-15 minutos.

Análisis de los datos

Se presentan descriptivos básicos. La distribución de las variables resultó normal a través de una prueba de normalidad de Kolmogorov-Smirnov. Las diferencias según sexo fueron analizadas mediante un análisis de la covarianza (ANCOVA) ajustado por sexo para las variables continuas, y el test de chi-cuadrado para las variables categóricas. Las diferencias de salud mental en función del estado de peso y el nivel de CF fueron analizadas mediante una prueba ANOVA, sin análisis post hoc. Se empleó el programa SPSS (v.19.0 de SPSS Inc., Chicago, IL, EE.UU.) fijándose el nivel de significación en $p<0,05$.

\section{RESULTADOS}

En la tabla 2 se presentan los descriptivos básicos de los parámetros del estudio. La prevalencia de escolares con sobrepeso-obesidad fue del 40,7\%, mientras que la prevalencia de escolares con $m$ CF- ALPHA fue del 39,7\%. Por su parte, la prevalencia de sospecha de psicopatología subumbral e indicativo de presencia de psicopatología para el total de la muestra fue del $10,3 \%$. 
El análisis ANCOVA detectó diferencias estadísticamente significativas en el test de $4 \times 10 m(p=0,002)$, salto longitudinal $(p=0,020)$, CourseNavette, y en el valor global de la CF (zCF-ALPHA; $p<0,001$, para ambos) a favor de los varones. No se detectaron diferencias en el resto de variables. El test de chi-cuadrado detectó diferencias significativas en el grupo de menor CF global ( $m C F-A L P H A)$, siendo superior su prevalencia en las mujeres $(p<$ 0,001 ). No se detectaron diferencias significativas en la prevalencia de salud mental (GHQ-12) en función del sexo, aunque si se observó una tendencia hacia la significación estadística en el grupo indicativo de presencia de psicopatología a favor de las mujeres $(p=0,083)$.

Tabla 2. Parámetros de estudio.

\begin{tabular}{|c|c|c|c|c|}
\hline & \multicolumn{4}{|c|}{ Sexo } \\
\hline & $\begin{array}{c}\text { Total } \\
(\mathrm{n}=214)\end{array}$ & $\begin{array}{l}\text { Varones } \\
(n=94)\end{array}$ & $\begin{array}{l}\text { Mujeres } \\
(n=120)\end{array}$ & \\
\hline Peso (kg) & $38,5 \pm 11,3$ & $38,5 \pm 11,6$ & $38,4 \pm 11,1$ & 0,996 \\
\hline Talla $(\mathrm{cm})$ & $140,0 \pm 12,8$ & $139,9 \pm 12,6$ & $140,0 \pm 12,9$ & 0,962 \\
\hline $\operatorname{IMC}\left(\mathrm{kg} / \mathrm{m}^{2}\right)^{\mathrm{a}}$ & $19,3 \pm 3,7$ & $19,2 \pm 3,5$ & $19,3 \pm 3,8$ & 0,864 \\
\hline Normopeso (\%) & 59,3 & 60,6 & 58,3 & 0,249 \\
\hline Sobrepeso (\%) & 29,0 & 30,9 & 27,5 & 0,611 \\
\hline Obesidad (\%) & 11,7 & 8,5 & 14,2 & 0,072 \\
\hline $4 \times 10 \mathrm{~m}(\mathrm{~s})$ & $13,7 \pm 1,4$ & $13,5 \pm 1,4$ & $13,9 \pm 1,4$ & 0,002 \\
\hline Dinamometría (kg) & $15,9 \pm 5,4$ & $16,0 \pm 5,8$ & $15,8 \pm 5,1$ & 0,261 \\
\hline Salto longitudinal $(\mathrm{cm})$ & $102,3 \pm 20,9$ & $105,5 \pm 20,2$ & $99,7 \pm 21,1$ & 0,020 \\
\hline $\mathrm{C}-\mathrm{N}$ (paliers) ${ }^{\mathrm{b}}$ & $3,3 \pm 1,7$ & $3,8 \pm 1,9$ & $2,9 \pm 1,3$ & $<0,001$ \\
\hline ZCF-ALPHA $^{\mathrm{c}}$ & $0,7 \pm 0,4$ & $0,8 \pm 0,4$ & $0,6 \pm 0,4$ & $<0,001$ \\
\hline mCF-ALPHA (\%) ${ }^{c}$ & 39,7 & 27,7 & 49,2 & $<0,001$ \\
\hline MCF-ALPHA (\%) ${ }^{c}$ & 60,3 & 72,3 & 50,8 & 0,538 \\
\hline GHQ-12 ${ }^{d}$ & $2 \pm 2$ & $2 \pm 2$ & $2 \pm 2$ & 0,210 \\
\hline Ausencia $(\%)^{d}$ & 89,7 & 91,5 & 88,3 & 0,149 \\
\hline Sospecha (\%) ${ }^{d}$ & 4,7 & 5,3 & 4,2 & 1,000 \\
\hline Indicativo $(\%)^{d}$ & 5,6 & 3,2 & 7,5 & 0,083 \\
\hline $\begin{array}{l}\text { Los resultados se pr } \\
\text { ANCOVA para varia } \\
\text { categóricas. a Índice } \\
\text { condición física en pu } \\
\text { y MCF-ALPHA = may } \\
\text { mental: ausencia de } \\
\text { indicativo de presenci }\end{array}$ & $\begin{array}{l}\text { aciones Z; } \\
\text { hivel de zCF } \\
\text { opatología, } \\
\text { e psicopatol }\end{array}$ & $\begin{array}{l}\text { edia } \pm \text { des } \\
\text { test de c } \\
{ }^{\text {b }} \text { Course-I } \\
\text { FALPHA = } \\
\text { PHA. d Sa } \\
\text { specha de } \\
\text { a. }\end{array}$ & $\begin{array}{l}\text { ión estánda } \\
\text { uadrado pa } \\
\text { tte; ' }{ }^{c} \text { Valor } \\
\text { or nivel de } \\
\text { nental; Nive } \\
\text { ppatología s }\end{array}$ & $\begin{array}{l}\text { Test de } \\
\text { variables } \\
\text { bal de la } \\
=- \text { ALPHA, } \\
\text { de salud } \\
\text { umbral, e }\end{array}$ \\
\hline
\end{tabular}


La tabla 3 muestra los resultados del análisis de la relación entre zCFALPHA y salud mental (GHQ-12). La prueba ANOVA no detectó diferencias estadísticamente significativas en la salud mental de los escolares en función del nivel de CF $(F=0,013 ; p=0,909)$. No obstante, se puede observar que los escolares con MCFALPHA presentaron en valor absoluto una mejor media de salud mental.

Tabla 3. Relación entre condición física y salud mental.

\begin{tabular}{ccccc}
\hline & \multicolumn{5}{c}{ GHQ-12 $^{\sqrt{ }}$} \\
& $\mathrm{N}$ & $\mathrm{M} \pm \mathrm{DE}$ & Error típico & IC 95\% LI-LS \\
\hline mCF-ALPHA $^{\dagger}$ & 85 & $1,7 \pm 2,0$ & 0,2 & $1,3-2,2$ \\
MCF-ALPHA $^{\dagger}$ & 129 & $1,8 \pm 2,1$ & 0,1 & $1,4-2,1$ \\
\hline
\end{tabular}

Los resultados se presentan como media \pm desviación estándar. mCFALPHA $=$ menor nivel de zCF-ALPHA, y MCF-ALPHA = mayor nivel de zCF-ALPHA; Salud mental.

Con respecto a la relación entre estado de peso y salud mental, los resultados encontrados se describen en la tabla 4. La prueba ANOVA tampoco detectó diferencias estadísticamente en la salud mental en función del estado de peso $(F=0,509 ; p=0,602)$. Sin embargo, los escolares con obesidad presentaron en valor absoluto una mejor media de salud mental.

Tabla 4. Relación entre estado de peso y salud mental (GHQ-12).

Estado de GHQ-12

\begin{tabular}{lcccc}
\cline { 2 - 5 } peso & $\mathrm{N}$ & $\mathrm{M} \pm \mathrm{DE}$ & Error típico & IC 95\% LI-LS \\
\hline Normopeso & 127 & $1,7 \pm 2,1$ & 0,1 & $1,4-2,1$ \\
Sobrepeso & 62 & $1,7 \pm 1,9$ & 0,2 & $1,2-2,2$ \\
Obesidad & 25 & $2,2 \pm 2,2$ & 0,4 & $1,2-3,1$
\end{tabular}

Los resultados se presentan como media \pm desviación estándar. ${ }^{\downarrow}$ Salud mental.

\section{DISCUSIÓN}

De la realización de este estudio se desprenden los siguientes hallazgos: i) no existe una asociación positiva entre la CF (expresada en este trabajo mediante la relación entre fuerza de prensión manual, potencia explosiva del tren inferior, capacidad aeróbica y velocidad-agilidad) y el estado de peso (categorizado a partir del IMC) con la salud mental; ii) los varones presentan un mejor nivel de CF, sobre todo, a nivel de velocidad-agilidad, potencia explosiva del tren inferior y capacidad aeróbica; iii) la prevalencia de mCF-ALPHA $\left(<\mathrm{P}_{50}\right)$ para el total de la muestra fue del $39,7 \%$; iv) la prevalencia de sobrepeso-obesidad del total de la muestra fue del $40,7 \% 8$; v) la 
prevalencia de escolares con sospecha o indicativo de psicopatología fue del $10,3 \%$ (4,7 y $5,6 \%$, respectivamente).

La mayoría de los estudios que han analizado desde el ámbito de la psicología de la actividad física y el deporte la relación entre CF y salud mental, se han centrado en la variable actividad físico-deportiva del individuo como hábito de estilo de vida determinante para un estado óptimo de bienestar ${ }^{20-24}$. Sin embargo, la CF es el predictor biológico con mayor relevancia del estado de salud y calidad de vida del individuo desde la infancia ${ }^{9,11}$, de ahí el enfoque novedoso de esta investigación.

Los escasos trabajos que han analizado la relación entre CF y salud mental muestran resultados contradictorios. En el presente estudio, los resultados muestran la inexistencia de una asociación consistente entre CF y salud mental en escolares de 8 a 11 años del sureste español, lo que concuerda con lo encontrado por Quin et al. ${ }^{27}$, en 311 escolares británicos de 11-14 años, tras analizar qué efectos tenía mejorar el estado de CF mediante la realización durante 10 semanas de ejercicio físico, sobre la salud mental.

Estos hallazgos también han sido observados en adultos. En este sentido, en un estudio se observó en 9 adultos con enfermedades mentales (depresión, enfermedades bipolares, esquizofrenia, y ansiedad) que la mejora de la CF general solo producía mejoras muy leves en el estado de ansiedad y depresión ${ }^{28}$. Por su parte, Selman et al. ${ }^{29}$ en 18 adultos con cáncer observaron tras la intervención realizada, una disminución en la variable preocupaciones pero no una mejora significativa en el bienestar autopercibido.

De la realización de estos estudios se podría concluir que la CF no constituye un factor relevante para la salud mental, por lo que futuras investigaciones deberían indagar en aquellos parámetros del estilo de vida o del entorno sociocultural que sean más determinantes.

Sin embargo, los resultados de otros trabajos realizados con escolares y personas jóvenes de Suecia y Estados Unidos difieren en este sentido, y apuntan a diversos parámetros de la CF, así como la CF general como determinantes de la salud mental en individuos desde edades tempranas ${ }^{25-26}$.

En la misma línea, Becerra y cols. ${ }^{18}$ en un trabajo realizado con 264 adolescentes de 14 a 16 años, en el cual estudiaron la asociación entre CF (medida a través de tres pruebas de la batería EUROFIT, carrera de $50 \mathrm{~m}$, salto horizontal y Course-Navette) y salud mental (medida a mediante la escala General Health Questionnaire-28) observaron que la CF se encontraba fuertemente asociada con la salud mental. Además, estos autores encontraron que algunos componentes de la CF como la capacidad aeróbica y la grasa corporal podían incluso predecir ciertas dimensiones de la salud mental tales como los síntomas somáticos y ansiedad e insomnio.

En adultos, uno de los escasos trabajos y con resultados más relevantes es el realizado por Jeoung y cols. ${ }^{19}$ con 228 universitarios coreanos (137

38 RELACIÓN ENTRE CONDICIÓN FÍSICA Y SALUD MENTAL EN ESCOLARES DE PRIMARIA 
mujeres) donde analizaron la relación entre la CF, medida a través de diversas pruebas de la batería Eurofit (dinamometría manual, test de Course-Navette, push-ups, sit-ups, sit and reach test e índice de masa corporal) y el estado de peso (categorizado a partir del IMC) con la salud mental, evaluada mediante un instrumento de auto-informe formado por 47 ítems que miden dimensiones específicas de la salud mental negativa (somatización, psicosis, paranoia, fobia, hostilidad, ansiedad, depresión, sensibilidad interpersonal y obsesión compulsiva). Los análisis de regresión lineal realizados mostraron una consistente asociación entre los distintos componentes de la CF (a excepción de la flexibilidad) y el estado de peso (obesidad), con las dimensiones de la salud mental negativa.

Estos datos no concuerdan con los observados en el presente estudio, a pesar emplear una metodología de estudio similar. Sin embargo, la edad de los participantes como factor principal y el entorno sociocultural de los mismos puede haber generado la diferencia en los resultados entre este estudio y los encontrados en la presente investigación.

Este trabajo no se encuentra exento de diversas limitaciones, las que cuales se derivan de la metodología de estudio (diseño de investigación y muestral, empleo de medidas de autoinforme). Este hecho hace que los resultados deban ser interpretados con precaución, además de no permitir la extrapolación de los mismos a una población estándar. Sería necesario considerar estas cuestiones en futuras investigaciones. Una de las fortalezas radica en la apertura de una línea de investigación, en la que se emplea a la CF como variable independiente en el estudio de la salud mental en escolares de primaria. Además, se evaluó la CF mediante una batería cuya validez, confiabilidad, y relación con la salud ha sido demostrada en escolares ${ }^{7,32}$.

\section{CONCLUSIONES}

Los resultados del presente trabajo de investigación sugieren que no existe una asociación positiva entre la CF y el estado de peso con la salud mental en escolares de 8 a 11 años del sureste español. Estudios longitudinales y prospectivos deberán determinar el modo en el que la tendencia a poseer o no un mejor nivel CF, y un estado de peso saludable o no saludable, pueden afectar a la salud mental de los individuos a lo largo de su escolarización y en periodos vitales posteriores.

\section{REFERENCIAS}

1. Blair, S. N. (2009). Physical inactivity: the biggest public health problem of the 21st century. British Journal of Sports Medicine, 43(1), 1-2.

2. Babiss, L. A. y Gangwisch, J. E. (2009). Sports participation as a protective factor against depression and suicidal ideation in adolescents as mediated by self-esteem and social support. Journal of Developmental and Behavioral Pediatrics, 30(5), 376-384. 
3. Jiménez, M. G., Martínez, P., Miró, E. y Sánchez, A. I. (2008). Bienestar psicológico y hábitos saludables: ¿están asociados a la práctica de ejercicio físico? International Journal of Clinical and Health Psychology, 8(1), 185-202.

4. Panagiotopoulos, C., Ronsley, R., Al-Dubayee, M., Brant, R., Kuzeljevic, B., Rurak, E. y Masse, L. C. (2011). The Centre for Healthy WeightsShapedown BC: A Family-Centered, Multidisciplinary Program that Reduces Weight Gain in Obese Children over the Short-Term. International Journal of Environmental Research and Public Health, 8(12), 4662-4678.

5. Sánchez-López, M., Salcedo-Aguilar, F., Solera-Martínez, F., MoyaMartínez, P. Notario-Pacheco, B. y Martínez-Vizcaíno, V. (2009). Physical activity and quality of life in schoolchildren aged 11-13 years of Cuenca, Spain. Scandinavian Journal of Medicine and Science in Sports, 19, 879-884.

6. Garcia-Artero E, Ortega FB, Ruiz JR, Mesa JL, Delgado M, GonzalezGross $\mathrm{M}$, et al. [Lipid and metabolic profiles in adolescents are affected more by physical fitness than physical activity (AVENA study)]. Rev Esp Cardiol. 2007;60:581-588.

7. Ruiz JR, España-Romero V, Castro-Piñero J, Artero EG, Ortega FB, Cuenca-García M, et al. Batería ALPHA-Fitness: test de campo para la evaluación de la condición física relacionada con la salud en niños y adolescentes, Nutr Hosp 2011; 26(6): 1210-1214.

8. García-Cantó E., Pérez-Soto JJ, Rodríguez-García PL, Rosa A., LópezMIñarro PA, López-Villalba FJ. The relationship between segmental coordination, agility and physical activity in adolescents. Motriz, Rio Claro 2015; (2): 200-206,

9. Ortega FB, Ruiz JR, Castillo MJ, Sjöström M. Physical fitness in childhood and adolescence: a powerful marker of health. Int $\mathrm{J}$ Obes (Lond). 2008;32:1-11.

10. Ruiz JR, Castro-Pinero J, Artero EG, Ortega FB, Sjostrom M, Suni J, et al. Predictive Validity of Health-Related Fitness in Youth: A Systematic Review. Br J Sports Med. 2009.

11. Gálvez A, Rodríguez-García PL, García-Cantó E, Rosa A, Pérez-Soto, JJ, Tárraga ML, Tárraga PJ. Capacidad aeróbica y calidad de vida en escolares de 8 a 12 años. Clin Investig Arterioscler 2015; 27(5): 239-24.

12. Gálvez A, Rodríguez-García PL, Rosa A, García-Cantó E, Pérez-Soto J.J., Tárraga, PJ, Tárraga ML. Capacidad aeróbica, estado de peso y autoconcepto en escolares de primaria. Clin Investig Arterioscler 2016; 28(1), 1-8.

13. Gálvez A, Rosa A, García-Cantó E, Rodríguez-García PL, Pérez-Soto, $\mathrm{JJ}$, Tárraga ML, Tárraga PJ. Estado nutricional y calidad de vida relacionada con la salud en escolares el sureste español. Nutr Hosp 2015; 31(2), 737-743.

14. Jiménez-Moral JA, Zagalaz ML, Molero D, Pulido-Martos M, Ruiz JR. Capacidad aeróbica, felicidad y satisfacción con la vida en adolescentes españoles. Revista de Psicología del Deporte 2013; 22(2), 429-436.

15. Rodríguez-García, P.L., Gálvez, A., García-Cantó, E., Pérez-Soto, J.J., Rosa, A., Tárraga, L. y Tárraga, P.L. Relationship between the Self- 
Concept and Muscular Strength in Southern Spanish Children. J Psychol Psychother 2014; 5, 222.

16. Rodríguez-García PL, Tárraga L, Rosa A, García-Cantó E, Pérez-Soto JJ, Gálvez A, Tárraga P. Physical Fitness Level and Its Relationship with Self-Concept in School Children. Psychology 2014; 5, 2009-2017.

17. Labrie JW, Kenney SR, Lac A. The use of protective behavioral strategies is related to reduced risk in heavy drinking college students with poorer mental and physical health. J Drug Educ 2010; 40: 361-378.

18. Becerra C, Reigal RE, Hernández-Mendo A, Martín-Tamayo J. Relationship of physical fitness and body composition with self-rated health. RICYDE. International Journal of Sport Science 2013; 34(9): 305318.

19. Jeoung $B$, Hong $M$, Lee $Y$. The relationship between mental health and health-related physical fitness of university students. J Exerc Rehabil 2013; 9(6): 544-548.

20.Dishman RK, Hales DP, Pfeiffer KA, Felton G, Saunders R, Ward DS, Pate R R. Physical self-concept and self-esteem mediate cross-sectional relations of physical activity and sport participation with depression symptoms among adolescent girls. Health Psychology 2006; 25(3): 396407

21. González J, Garcés de los Fayos E, García A. Indicadores de bienestar psicológico percibido en alumnos de Educación Física. Revista de Psicología del Deporte 2012; 21(1): 183-187.

22. Jeong YJ, Hong SC., Lee MS, Park MC, Kim YK, Suh CM. Dance movement therapy improves emotional responses and modulates neurohormones in adolescents with mild depression. International Journal of Neuroscience 2005; 115(12): 1711-1720.

23. Mavrovouniotis FH, Argiriadou EA, Papaioannou CS. (2010). Greek traditional dances and quality of old people's life. J Bodyw Mov Ther 2010; 14(3): 209-218.

24. Philipsson A, Duberg A, Moller M, Hagberg L. Costutility analysis of a dance intervention for adolescent girls with internalizing problems. Cost Eff Resour Alloc 2013; 11(1), 4.

25. Duberg A, Hagberg L, Sunvisson H, Moller M. Influencing self-rated health among adolescent girls with dance intervention: a randomized controlled trial. JAMA Pediatr 2013, 167(1): 27-31.

26. Wagener TL, Fedele DA, Mignogna MR, Hester CN, Gillaspy SR. Psychological effects of dance-based group exergaming in obese adolescents. Pediatr Obes 2012; 7(5): e68-74.

27. Quin E, Frazer L, Redding E. The Health Benefits of Creative Dance: improving children's physical and psychological wellbeing. Education and health 2007; 25(2): 31-33.

28. Hackney ME, Earhart GM. Social partnered dance for people with serious and persistent mental illness: a pilot study. J Nerv Ment Dis 2010, 198(1): 76-78.

29. Selman LE, Williams J, Simms V. A mixed-methods evaluation of complementary therapy services in palliative care: yoga and dance therapy. Eur J Cancer Care (Engl) 2012; 21(1): 87-97. 
30. Cole TJ, Bellizzi MC, Flegal KM, Dietz WH. Establishing a standard definition for child overweight and obesity worldwide: international survey. BMJ 2000; 320:1240-3.

31. Vicente-Rodríguez G, Rey-López JP, Mesana MI, Poortvliet E, Ortega FB, Polito A, Moreno LA. Reliability and intermethodagreement for body fat assessment among two field and two laboratory methods in adolescents. Obesity 2012; 20(1): 221-228.

32. España-Romero V, Ortega FB, Vicente-Rodríguez G, Artero EG, Rey JP, Ruiz, JR. Elbow Position Affects Handgrip Strength in Adolescents: Validity and Reliability of Jamar, Dynex, and Tkk Dynamometers. J Strength Cond Res 2010; 24(1): 272-277.

33. Castro-Pinero J, Ortega FB, Artero EG, Girela-Rejon MJ, Mora J, et al. Assessing muscular strength in youth: usefulness of standing long jump as a general index of muscular fitness. Journal of Strength and Conditioning Research 2010; 24:1810-1817.

34. Léger LA, Mercier D, Gadoury C y Lambert J. The multistage 20 metre shuttle run test for aerobic fitness. J Sports Sci 1988; 6:93-101.

35. Goldberg DP, Williams P. Cuestionario de Salud General GHQ. Barcelona: Masson; 1996.

36. Goldberg DP, Gater R, Sartorius N, Ustun TB, Piccinelli M, Gureje O, Rutter C. The validity of two versions of the GHQ in the WHO study of mental health illness in general health care. Psychological Medicine 1997; 27:191-197.

37. Sánchez-López MP, Dresch V. The 12-Item General Health Questionnaire (GHQ-12): Reliability, external validity and factor structure in the Spanish population. Psicothema 2008; 20:839-843.

Referencias totales citadas: 37

Referencias citadas correspondientes a la Rev lb CC Act Fis Dep: 0 